\title{
Obtrusiveness of smartphone applications for sleep health
}

\author{
M. Vazquez-Briseno, A. Laflor-Hernandez, F. Murillo-Munoz, D. Mendez-Verdugo \\ Universidad Autonoma de Baja California \\ Facultad de Ingenieria, Arquitectura y Diseño, Mexico
}

\begin{tabular}{|c|c|}
\hline Article Info & ABSTRACT \\
\hline $\begin{array}{l}\text { Article history: } \\
\text { Received May 25, } 2018 \\
\text { Revised Sep 18, } 2018 \\
\text { Accepted Oct 2, } 2018 \\
\text { Keywords: } \\
\text { Mobile applications } \\
\text { Obtrusiveness } \\
\text { Sleep } \\
\text { Smart phones }\end{array}$ & $\begin{array}{l}\text { Unobtrusiveness is one of the main issues concerning health-related systems. } \\
\text { Many developers affirm that their systems do not burden users; however, this } \\
\text { is not always achieved. This article evaluates the obtrusiveness of various } \\
\text { systems developed to improve sleep quality. The systems analyzed are } \\
\text { related to sleep hygiene, since it has become an interesting topic for } \\
\text { researchers, physicians and people in general, mainly because it has become } \\
\text { part of the methods used to estimate a persons' health status A set of design } \\
\text { elements are presented as keys to achieving unobtrusiveness. We propose a } \\
\text { scale to measure the level of unobtrusiveness and use it to evaluate several } \\
\text { systems, with a focus on smartphone applications. } \\
\text { Copyright } \text { (C) } 2019 \text { Institute of Advanced Engineering and Science. } \\
\text { All rights reserved. }\end{array}$ \\
\hline $\begin{array}{l}\text { Corresponding Author: } \\
\text { Mabel Vazquez-Briseno, } \\
\text { Faculty of Engineering, Architecture } \\
\text { Autonomous University of Baja Cali } \\
\text { Transpeninsular 3917, Col Playitas. I } \\
\text { Email: mabel.vazquez@uabc.edu.x }\end{array}$ & $\begin{array}{l}\text { and Design, } \\
\text { ornia, } \\
\text { nsenada, B.C., Mexico. }\end{array}$ \\
\hline
\end{tabular}

\section{INTRODUCTION}

The phenomenon of sleep hygiene has aroused interest in recent years, mainly because it has become part of the methods used to estimate a persons' health status. Many people are interested in this area, even those that do not suffer sleep issues. One of the reasons is because of the relationship that researchers have found between poor sleep quality and various chronic degenerative diseases such as diabetes and Alzheimer's disease. Systems and technological devices have been proposed to deal with this phenomenon from the clinical and non-clinical points of view. In all cases, the systems are evaluated to measure their efficiency in achieving their purpose. According to [1] perceived usefulness is a factor that can influence user acceptance. However, we have found that understanding user perceptions of the use of such systems is also important for gaining a complete understanding of the success of their designs. In this sense, unobtrusiveness is as important in the field of user perception as is the evaluation of the efficiency of the technical system. Choe et al. [2] found that unobtrusive solutions that allow users to track their sleep own behaviors represent an area of tremendous latent opportunity. After interviews with sleep disorder specialists $(N=4)$, the authors in [2] found that it is possible to achieve a satisfactory trade-off between the accuracy and unobtrusiveness of sleep monitoring. Authors argue that systems designed to track sleep and make sleep disorder diagnoses should consider obtrusiveness as a system goal. On the other hand, polysomnography (PSG), the golden standard test for sleep disorders in the medical domain, is considered the most obtrusive test.

In recent years, actigraphy has been used clinically. It is less obtrusive than PSG and can monitor populations and disorders that are not suitable for PSG monitoring [3], [4]. Nonetheless, PSG and actigraphy are obtrusive, high-cost and not accessible tools to all users. Physicians agree and they see the obtrusiveness of devices as a limiting factor; however, they also see the technology as a viable option to attend to this area of health. In this context, new approaches to the phenomenon have emerged in recent years. Researchers 
have designed systems based on environment sensors, wearable devices and portable-PSGs. On the other hand, designers have developed a wide range of mobile applications to help people sleep better. Mobile applications are a new approach to deal with obtrusiveness due to the symbiosis between mobile devices and users.

In this paper, we analyze the unobtrusiveness concept in the context of technological systems designed to address sleep-related issues, specifically we address smartphone applications for sleep health. We found that currently, researchers refer to the unobtrusiveness concept by diverse terms. In this context we analyzed a diverse range of systems intended to help people sleep better. For the purposes of giving clarity to the unobtrusive characteristics of technological designs, we classify existing systems in terms of their unobtrusiveness. We propose a scale for measuring the level of unobtrusiveness according with the literature reviewed. We propose a 7-point scale using seven metrics to determine the level of unobtrusiveness of a system. Systems meeting all the criteria (7/7) are considered optimum in relation to obtrusiveness. Furthermore we present the features that a mobile system should comprise in order to be considered unobtrusive, these findings will provide valuable information about mobile applications for sleep health systems useful for developers, researchers, users, and decision makers.

The rest of the paper is organized as follows: In Section 2 we present the research method. In section 3, we describe and analyze several smartphone applications for sleep health. Section 4 presents the discussion and results, including a proposal for measuring the level of unobtrusiveness and features to achieve an unobtrusive mobile system. Finally, Section 5 summarizes this paper.

\section{RESEARCH METHOD}

We performed a literature review on sleep health systems for papers from 2011 onwards. We build queries for research databases using five keywords: "sleep", "health", "unobtrusiveness", "mobile" and "systems" and the synonymous of keywords when apply. The term "sleep" was restrictive and must be found in the title, the other terms would be found in title, keywords or abstract sections. We limited the find to English language and manuscripts dated from 2011 to present. Titles and abstracts were reviewed to eliminate papers not relevant to the research. When necessary, full text was analyzed. After this process, some articles were added based in the references of selected papers. A summary of each paper was performed explaining the most relevant topics developed by the authors.

We found that researchers referred to the concept of unobtrusive using different terms. Some authors used invasive as a synonym of obtrusive, and non-invasive for unobtrusive [5], [6]. We also identified the use of the ubiquitous term in the form of unobtrusiveness. Since ubiquitous computing can occur with any device, at any time, in any place, from our own point of view people can get easily used to interact with a ubiquitous device that acquires the quality of imperceptible, and therefore became unobtrusive. Cumbersome was a term used to refer to the concept of obtrusive; however, it was only used in extreme cases of obtrusiveness such as the PSG test [7]. Other authors prefer to use the term "no user burden" for unobtrusiveness. After analyzing the term unobtrusive in the context of technology applied to sleep, we conclude that to be considered unobtrusive, the technology must fulfill the function for which it was designed without being perceptible to the user. Unobtrusiveness refers to the fact that the user is not aware of the use of the technology. Also, technology is considered unobtrusive when it is part of the user's natural environment. It means that users can carry out their activities without feeling disturbed, monitored or distracted.

\section{APPROACHES TO UNOBTRUSIVE SYSTEMS FOR SLEEP HEALTH}

Many applications and devices have been developed to promote healthy sleep. Some of them sense sleep related factors such as room temperature, noise, among other factors [8][9][10]. Some others sense and measure sleep quality, while others discover sleep habits, and some do both. The systems may include wearable devices; sensors placed in various objects around the sleep environment, and smartphone applications. In this paper we analyze systems that involve using smartphones. Krishna et al. [11] argue that achieving high accuracy in sleep measurements has been prioritized over non-intrusiveness. They also imply that obtrusive has to do with wearable devices that are too engaging and track user activities continuously. In this scenario, mobile applications seem to be the least obtrusive devices. This is due to the familiarity that users already have with them, as smartphones and tablets have became ubiquitous devices in recent years. The presence of these devices next to the users at night is useful for achieving the main goals of their designers. The following subsections describe the range of systems, which are classified in two categories: those that require users' conscious interaction, and those that do not. 


\subsection{Systems without user interaction}

With the aim of achieving unobtrusiveness, many studies have proposed the use of mobile phones that have sensors which can capture relevant clinical data in real time [12]. These technologies are considered unobtrusive because the user does not interact with sensors in the mobile phone. For instance, the application (app) iSleep uses the microphone of a smartphone to infer the quality of sleep Error! Reference source not found. The app precisely identifies acoustic signals to detect the sleep-related events of the user. In a similar approach, [13] uses the built-in microphone of a smartphone to listen to breathing sounds to estimate sleep quality. The app BES (Best effort sleep) [15] infers sleep duration by tracking the status and sensors of a smartphone, such as being locked, on/off or charging, and sensing light levels, movement and ambient noise. Contrary to the iSleep approach, in BES, no audio is recorded to guarantee privacy to the users.[16] Also uses the sensors in the smartphone to track daily sleep patterns. Another application using a different perspective

SleepCoacher [17]. This system collects the raw data of mobile phone sensors and generates personalized data-driven sleep recommendations that are generated by recognizing connections between sleep behaviors and sleep outcomes; the recommendation text comes from a collection of templates generated with the help of clinicians. An alternative approach based in the concept of sleep hygiene was implemented in SleepMiner [18]. This app extracts several features such as daily activity, living environment and social activity from mobile phone data. Sleep quality is next predicted by an algorithm that uses data collected by the smartphone's sensors.

Other approaches for measuring the quality of sleep and for monitoring sleep patterns use sensors in devices such as bracelets, headbands and cameras, or devices built for specific purposes. For instance, ubiSleep [19] uses two devices to monitor sleep quality: a smart-watch to collect the data and a smartphone to display the results. ubiSleep consider both devices to be ubiquitous. Consequently, the complete system is qualified as unobtrusive. J. Paalasmaa et al. [20] and Papakostas [21] use a similar approach to obtain sleep data via sensors placed in the bed. Processed data is displayed to the user by a mobile device. Paalasmaa and his colleagues [20] proposed the Beddit system to estimate variables such as sleep quality, sleep latency and effectiveness of sleep. To measure these factors, Beddit uses a thick band placed under the bed sheet to monitor heart rate, breathing, and posture motion. Additionally, Beddit uses smartphone sensors to collect data such as luminosity and noise. On the other hand, Papakostas et al. [21] use a mattress with an array of pressure sensors that monitor the user's position, while a video camera monitors breathing by detecting chest movement.

\subsection{Systems with user interaction}

Other kind of systems developed to attend to issues related to sleep behaviors require some level of user interaction. However, researchers affirm that even when users interact explicitly with a technology, it is an unobtrusive activity. Some systems interact with users during daylight, such as ShutEye [22], an application that encourages users to be conscious of sleep hygiene. ShutEye uses a wallpaper with different colored bars representing its recommendations. The app assumes that users are able to perceive these tips at a glance, due to the design of the interface. In addition, the information is available when needed and easily ignored otherwise, causing minimal user burden. Another app that interacts with users in daytime, but more dynamically than ShutEye, is Toss 'N' Turn [23]. It collects sensor data regarding the number of apps running on the smartphone, its battery level and screen state. Additionally, Toss ' $N$ ' Turn asks people to fill out a daily sleep diary and uploads the data daily to a server.

In a different approach, some apps interact with users at night. Their designers argue that these apps are unobtrusive, for example, [24] and [25]. [25] Proposes SleepfulApp, a smartphone app designed to measure sleep efficiency. It requires less user interaction than ShutEye; nonetheless, interaction is required at critical moments. Users must start the action of recording when they go to bed and stop it after waking up. Furthermore, users must interact with the application each time that they get out of bed and came back to it during the night. The most critical interaction is generated by the system in the process of obtaining data used to compute sleep duration. To determine the time when the user fell asleep, the system emits low volume audio tones and waits for a user response. The last response indicates the time at which the user fell asleep. The time when the user wakes up is indicated by the moment at which the user stops the application in the morning. Another app in the same paradigm of SleepfulApp is SleepSensei, [11], which is a sleep duration estimator designed to be unobtrusive. SleepSensei emits an auditory tone every 15 minutes during the sleep period to find out whether the user is in deep or light sleep. After these tones, the system tracks the movements of the user over the following two minutes to determine the depth of sleep. SleepSensei claims unobtrusiveness because it avoids user acquisition of additional equipment and requires minimal user engagement. 
In a different approach, Lullaby [26] and EZwakeup [27] use a set of heterogeneous sensors around the bed to get sleep-related data with little or no user interaction. One of the most objectionable things about these projects is that additional infrastructure is necessary to implement the systems. Additionally, in Lullaby the user must wear a wrist device to measure sleep time according to the pressing of a button before and after the sleep period. Similar, in EZwakeup an eTextile sensor sheet needs to be placed underneath the user's existing bed sheet. For people suffering sleep disorders, these devices are obtrusive.

\section{RESULTS AND DISCUSSION}

Based on our literature review of sleep systems and user perceptions of them we found that people are likely to reject technology that disturbs their daily activities or requires additional daily work (e.g. manually capturing of data). Likewise, users prefer systems that do not need additional technology to be implemented. People with sleep disorders often disagree to wear a wrist or head device and even some users consider a camera to be an intrusive sensor. Furthermore, experts do not recommend technology that separates users from their natural behavior patterns or their natural environment, e.g., they do not recommend alarms as they cause people to wake because of external intervention rather than naturally. In consideration of these user opinions, and researchers opinions the best approach for unobtrusiveness is to remove users completely from the monitoring process and not require any interaction with systems or applications. Based on our analysis we have defined five scenarios with different level of obtrusiveness in order to classify sleep monitoring systems, as shown in Figure 1.

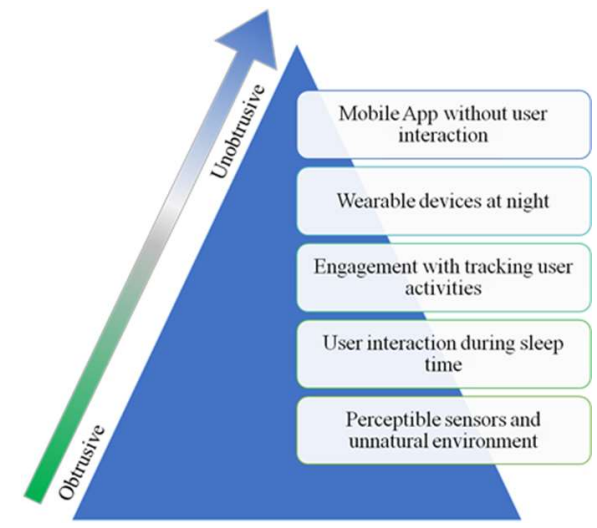

Figure 1. Obtrusiveness scenarios of sleep monitoring systems

In the bottom of the Figure 1 is the most obtrusive scenario. It requires people to sleep in an unnatural environment and wear perceptible sensors on their body, such as in the PSG test. On the other hand, at the top of the Figure 1 is the category for mobile apps that need no user interaction. We consider systems that require user interaction at night to be more obtrusive than those that only require interaction during the day. Applications using wearable devices offer an interesting scenario to analyze. In general terms, healthy people do not consider these sensors obtrusive, while people suffering sleep disorders do. Finally, independently of the researchers' arguments, the level of obtrusiveness in all systems is determined by users, using a subjective evaluation. Furthermore, we propose seven features to measure the unobtrusiveness of systems designed for sleep health, and classify them as shown in Table 1. The features included are:

a. Perceptible sensors: Is the user aware of the presence of sensors? Can they feel them?

b. Input mechanisms: Classified as Automatic/By user/Both/None. Automatic means that the system collects information without user interaction, probably by sensors. Manually means that users enter information through a GUI, or by using some mechanism that requires time and concentration. This could be annoying to users because it interrupts their daily activities.

c. User interaction: This measure refers to the fact that users are aware of the system's activity. Additionally, the users must enter some data to the system. Does the user have to dedicate time to the application? Should the user enter any data when the system requests it? Possible categories, indicating interaction/timing are: [High-Night/Low-Night/Low-Day/High-Day/Low-Both/High-Both]."HighBoth" is the most obtrusive qualification and "Low-Day" is the least.

d. Engagement: The possible answers are [Yes/No]. "Yes" means that systems were designed with the intention of encouraging users to use the system as much as possible. 
e. Wearable: The four categories [Day/Night/Both/None] indicate whether users must wear a device at night, during the day, both, or not at all.

f. Tracking user activities continuously: This concept refers to those activities that users do during the day. It does not include the sensing of sleep patterns at night. Does the user have to give information to the application during the day? The possible answers are [Yes/ No].

g. Natural environment: Does the user have to move to another place to be monitored by the system or can they sleep in their natural home environment? Possible answers are [Yes/No]

Table 1. Obtrusiveness of Various Sleep Systems

\begin{tabular}{|c|c|c|c|c|c|c|c|}
\hline System & $\begin{array}{c}\text { Perceptible } \\
\text { sensors }\end{array}$ & $\begin{array}{c}\text { Input } \\
\text { mechanism }\end{array}$ & $\begin{array}{c}\text { User } \\
\text { interaction }\end{array}$ & Engagement & Wearable & $\begin{array}{c}\text { Tracking user } \\
\text { activities }\end{array}$ & $\begin{array}{c}\text { Natural } \\
\text { environment }\end{array}$ \\
\hline Hao et al. [13] & No & Automatic & None & No & None & No & Yes \\
\hline Chen et al. [15] & No & Automatic & None & No & None & Yes & Yes \\
\hline Fahim et al. [16] & No & Automatic & None & No & None & No & Yes \\
\hline Daskalova et al. [17] & No & Automatic & None & No & None & No & Yes \\
\hline Paalasmaa et al. [20] & No & Automatic & Low-Night & No & None & Yes & Yes \\
\hline Papakostas et al. [21] & Yes & Automatic & None & No & None & Yes & Yes \\
\hline Bauer et al. [22] & No & None & None & No & None & No & Yes \\
\hline Min et al. [23] & No & Both & High-Day & No & None & Yes & Yes \\
\hline Lawson et al. [24] & Yes & By user & Low-Night & No & None & No & Yes \\
\hline
\end{tabular}

We can see that all of the systems in Table 1 satisfy at least 4 of the 7 criteria of unobtrusiveness. This is a good result compared with the old PSG test, which only meets $2 / 7$ criteria (engagement and input mechanisms). Based on Figure 1, we conclude that the most obtrusive systems are the ones proposed in [14], [23] and [24] due to users sleeping in an unnatural environment with perceptible sensors. On the other hand, [13], [16], [17] and [27] are systems that can be considered unobtrusive. We are aware that the nature and goals of the systems are different; however, we are only comparing unobtrusiveness, not system efficiency, which is the other half of an evaluation. We summarize the unobtrusiveness levels of analyzed systems in Table 2.

Once we analyzed the desirable features of sleep health systems, we present a scenario of a perfectly unobtrusive system in Table 3. We considered that systems should not used perceptible sensors. All inputs have to be automatic. Users should not be aware of the system, this includes that the users should not feel he needs to use the system, even though this could be a desirable feature in some other type of applications. Finally, the system does not require a wearable device, it does not track activities during the day and the user can sleep in a natural environment.

Table 2. Classification of Sleep Monitoring Systems by Level of Unobtrusiveness

\begin{tabular}{llll}
\hline \multicolumn{1}{c}{ Unobtrusive (7/7) } & \multicolumn{1}{c}{ Low Obtrusiveness (6/7) } & Medium Obtrusiveness (5/7) & Obtrusive (4/7) \\
\hline Hao et al. [13] & Bai et al. [18] & Krishna et al. [11] & Min et al. [23] \\
Fahim et al. [16] & Bauer et al. [22] & Chen et al. [15] & Lawson et al. [24] \\
Daskalova et al. [17] & & Pombo \& Garcia [19] & \\
Huang et al. [27] & & Paalasmaa et al. [20] & \\
& Papakostas et al. [21] & \\
& Han et al. [25] & Kay et al. [26] \\
\hline
\end{tabular}

Table 3. Best Scenario for Unobtrusiveness

\begin{tabular}{ll}
\hline Feature & Best option \\
\hline Perceptible sensors & No \\
Input mechanisms & Automatic/None \\
User interaction & No \\
Engagement & No \\
Wearable & No \\
Tracking user activities continuosly & No \\
Natural environment & Yes \\
\hline
\end{tabular}

Int J Elec \& Comp Eng, Vol. 9, No. 1, February 2019: 676 - 682 


\section{CONCLUSION}

Concluding, we qualify unobtrusiveness as a desirable feature in sleep technology. However, it is a subjective feature that depends of various factors that influence the level of perceived obtrusiveness. Among the most significant factors are the following: 1) presence of sleep disorders; 2) knowledge of how the technology can help; 3) awareness of the harm of poor-quality sleep. We provide a set of features that can be used to measure the level of obtrusivennes of mobile applications developed for sleep health. We define the best scenario for an unobtrusive application. Additionally, we found that the high cost of technology is considered to be a user burden. In smartphone applications, users perceive no cost burden, because no additional hardware is involved. In healthy people with intentions to improve quality of life, commercial devices and smartphones applications are welcome. Unobtrusiveness comes down to a cost-benefit trade-off.

\section{REFERENCES}

[1] T. Prayoga,, \&, J. Abraham, "Behavioral Intention to Use IoT Health Device: The Role of Perceived Usefulness, Facilitated Appropriation, Big Five Personality Traits, and Cultural Value Orientations". International Journal of Electrical and Computer Engineering (IJECE), 6(4), pp 1751-1765 ISSN: 2088-8708, 2016.

[2] E. K. Choe, S. Consolvo, N. F. Watson, and J. A. Kientz, "Opportunities For Computing Technologies To Support Healthy Sleep Behaviors," in Proceedings of the SIGCHI Conference on Human Factors in Computing Systems, ser. CHI '11. New York, NY, USA: ACM, 2011, pp. 3053-3062. [Online]. Available: http://doi.acm.org/10.1145/1978942.1979395, 2011.

[3] A. Sadeh, "The Role and Validity of Actigraphy in Sleep Medicine: An Update," Sleep Medicine Reviews, vol. 15, no. 4, pp. 259-267, 2011.

[4] V. Natale, D. Lger, M. Martoni, V. Bayon, and A. Erbacci, "The Role of Actigraphy in the Assessment of Primary Insomnia: A Retrospective Study,” Sleep Medicine, vol. 15, no. 1, pp. 111-115, 2014.

[5] D. Gartenberg, R. Thornton, M. Masood, D. Pfannenstiel, D. Taylor, and R. Parasuraman, "Collecting HealthRelated Data on the Smart Phone: Mental Models, Cost of Collection, And Perceived Benefit of Feedback," Personal and Ubiquitous Computing, vol. 17, no. 3, pp. 561-570, 2013.

[6] V. Metsis, D. Kosmopoulos, V. Athitsos, and F. Makedon, "Noninvasive Analysis Of Sleep Patterns Via Multimodal Sensor Input," Personal and Ubiquitous Computing, vol. 18, no. 1, pp. 19-26, 2014.

[7] E. K. Choe, "Design Of Persuasive Technologies For Healthy Sleep Behavior," in Proceedings of the 13th International Conference on Ubiquitous Computing, ser. UbiComp '11. New York, NY, USA: ACM, pp. 507-510, 2011.

[8] M. Borazio and K. Van Laerhoven, "Combining Wearable And Environmental Sensing Into An Unobtrusive Tool For Longterm Sleep Studies," in Proceedings of the $2^{\text {nd }}$ ACM SIGHIT International Health Informatics Symposium, ser. IHI '12. New York, NY, USA: ACM, 2012, pp. 71-80,2012.

[9] Bin Mohd Saad, W. H., Wuen, K. C., bin Mat Ibrahim, M., Saad, N. H. B. M., Radz, S. B. A., bin Mohamad Shokri, A. S.,, \& bin Karis, M. S. "Study on the Effect of the Ambient Temperature toward the Quality of Sleep". International Journal of Electrical and Computer Engineering (IJECE), 7(6), 2986-2992, 2017.

[10] Zhou, H., He, X., Yang, J., \& Liao, L. "Design of Remote Control Juvenile Healthy Smart Pillow". Indonesian Journal of Electrical Engineering and Computer Science, 7(1), 97-106, 2017.

[11] A. Krishna, M. Mallick, and B. Mitra, "Sleepsensei: An Automated Sleep Quality Monitor And Sleep Duration Estimator," in Proceedings of the First Workshop on IoT-enabled Healthcare and Wellness Technologies and Systems, ser. IoT of Health '16. New York, NY, USA: ACM, 2016, pp. 29-34 [Online]. Available: http://doi.acm.org/10.1145/2933566.2933570, 2016.

[12] C. N. Kaufmann, A. Gershon, L. T. Eyler, and C. A. Depp, "Clinical Significance Of Mobile Health Assessed Sleep Duration And Variability In Bipolar Disorder," Journal of Psychiatric Research, vol. 81, pp. 152- 159, 2016.

[13] T. Hao, G. Xing, and G. Zhou, "Isleep: Unobtrusive Sleep Quality Monitoring Using Smartphones," in Proceedings of the 11th ACM Conference on Embedded Networked Sensor Systems. ACM, p. 4, 2013.

[14] Y. Ren, C. Wang, J. Yang, and Y. Chen, "Fine-Grained Sleep Monitoring: Hearing Your Breathing with Smartphones," in 2015 IEEE Conference on Computer Communications (INFOCOM), April 2015, pp. 1194-1202.

[15] Z. Chen, M. Lin, F. Chen, N. D. Lane, G. Cardone, R. Wang, T. Li, Y. Chen, T. Choudhury, and A. T. Campbell, "Unobtrusive Sleep Monitoring Using Smartphones," in 2013 7th International Conference on Pervasive Computing Technologies for Healthcare and Workshops, pp. 145-152, May 2013.

[16] M. Fahim, L. B. Vui, I. Fatima, S. Lee, and Y. Yoon, "A Sleep Monitoring Application for u-lifecare using Accelerometer Sensors of Smartphones," Cham: Springer International Publishing, pp. 151- 158, 2013.

[17] N. Daskalova, D. Metaxa-Kakavouli, A. Tran, N. Nugent, J. Boergers, J. McGeary, and J. Huang, "Sleepcoacher: A Personalized Automated Self-Experimentation System For Sleep Recommendations," in Proceedings of the 29th Annual Symposium on User Interface Software and Technology, ser. UIST '16. New York, NY, USA: ACM, pp. 347-358. [Online]. Available: http://doi.acm.org/10.1145/2984511.2984534, 2016.

[18] Y. Bai, B. Xu, Y. Ma, G. Sun, and Y. Zhao, "Will You Have A Good Sleep Tonight? Sleep Quality Prediction with Mobile Phone," in Proceedings of the 7th International Conference on Body Area Networks, ser. BodyNets '12. ICST, Brussels, Belgium. ICST (Institute for Computer Sciences, Social-Informatics and Telecommunications Engineering, pp. 124-130. [Online]. Available: http://dl.acm.org/citation.cfm?id=2442691.2442720), 2012. 
[19] N. Pombo and N. M. Garcia, "Ubisleep: A Ubiquitous Sensor System For Sleep Monitoring," in 2016 IEEE 12th International Conference on Wireless and Mobile Computing, Networking and Communications (WiMob), Oct 2016, pp. 1-4.

[20] J. Paalasmaa, T. Sarkanen, and M. Partinen, "Long-Term Sleep Measurement With A Smartphone-Connected Flexible Bed Sensor Strip," Sleep Medicine, vol. 14, p. e258, 2013.

[21] M. Papakostas, J. Staud, F. Makedon, and V. Metsis, "Monitoring Breathing Activity And Sleep Patterns Using Multimodal Noninvasive Technologies," in Proceedings of the 8th ACM International Conference on Pervasive Technologies Related to Assistive Environments, ser. PETRA '15. New York, NY, USA: ACM, 2015, pp. 78:178:4. [Online]. Available: http://doi.acm.org/10.1145/2769493.2769585

[22] J. S. Bauer, S. Consolvo, B. Greenstein, J. Schooler, E. Wu, N. F. Watson, and J. Kientz, "Shuteye: Encouraging awareness of healthy sleep recommendations with a mobile, peripheral display," in Proceedings of the SIGCHI Conference on Human Factors in Computing Systems, ser. CHI '12. New York, NY, USA: ACM, pp. 1401-1410, 2012.

[23] J.-K. Min, A. Doryab, J. Wiese, S. Amini, J. Zimmerman, and J. I. Hong, "Toss 'N' Turn: Smartphone As Sleep And Sleep Quality Detector," in Proceedings of the SIGCHI Conference on Human Factors in Computing Systems, ser. CHI '14. New York, NY, USA: ACM, pp. 477-486. [Online]. Available: http://doi.acm.org/10.1145/2556288.2557220, 2014

[24] S. Lawson, S. Jamison-Powell, A. Garbett, C. Linehan, E. Kucharczyk, S. Verbaan, D. A. Rowland, and K. Morgan, "Validating A Mobile Phone Application For The Everyday, Unobtrusive, Objective Measurement Of Sleep," in Proceedings of the SIGCHI Conference on Human Factors in Computing Systems, ser. CHI '13. New York, NY, USA: ACM, pp. 2497-2506. [Online]. Available: http://doi.acm.org/10.1145/2470654.2481345, 2013.

[25] Han, T. Y., Min, S. D., \& Nam, Y, “A real-time sleep monitoring system with a smartphone,” In Innovative Mobile and Internet Services in Ubiquitous Computing (IMIS), 2015 9th International Conference on (pp. 458-461). IEEE, July 2015.

[26] M. Kay, E. K. Choe, J. Shepherd, B. Greenstein, N. Watson, S. Consolvo, and J. A. Kientz, "Lullaby: A Capture Access System For Understanding The Sleep Environment," in Proceedings of the 2012 ACM Conference on Ubiquitous Computing, ser. UbiComp '12. New York, NY, USA: ACM, 2012, pp. 226-234, 2012.

[27] M.-C. Huang, X. Zhang, W. Xu, J. Liu, and M. Sarrafzadeh, "Ezwakeup: A Sleep Environment Design For Sleep Quality Improvement," in CHI '14 Extended Abstracts on Human Factors in Computing Systems, ser. CHI EA '14. New York, NY, USA: ACM, pp. 2389-2394. [Online]. Available: http://doi.acm.org/10.1145/2559206.2581250, 2014.

\section{BIOGRAPHIES OF AUTHORS}

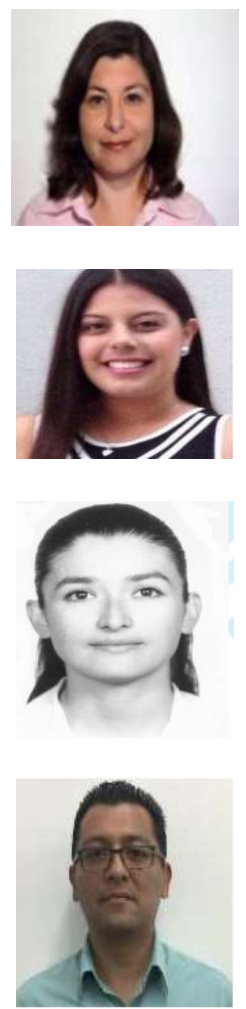

Mabel Vazquez-Briseno Received her PhD in Computer Science from Telecom SudParis (ex INT) and Pierre et Marie Curie University, France in 2008. She received the M.Sc degree in Electronics and Telecommunications from CICESE Research Center, Mexico, in 2001. She is now researcher-professor at the Autonomous University of Baja California (UABC), where she is a member of the Telematics research group. Her research interests include computer networks, mobile computing and protocols.

Fernanda Murillo-Muñoz is an MSc student at Autonomous University of Baja California. She completed her bachelor degree in Computers Engineering in 2015- Her research interests include: Interactive systems design, advances in mobile applications and persuasive systems.

Doreen Mendez is an Msc student at Autonomous University of Baja California. She completed her bachelor degree in Computers Engineering in 2000. Her research interests include: smartphones sensors focused in biosignals.

Arturo Laflor-Hernandez is a $\mathrm{PhD}$ student at Autonomous University of Baja California. He completed his master degree in Distributed and Embedded Systems in 2010 with the thesis Crosslayer protocol to communicate a Wireless sensor network on 802.15.4. Currently he designs an adaptative model to estimate the quality of sleep hygiene using machine learning algorithms. His research interests include: Machine learning, mobile HCI and bioengineering. 